\title{
Calculation of capacitances of silicon micro-strip sensors with a two dimensional numerical solution of the Laplace's equation
}

\author{
P. Assiouras ${ }^{* a}$, P. Asenov ${ }^{a}$, I. Kazas ${ }^{a}$, A. Kyriakis ${ }^{a}$ and D. Loukas ${ }^{a}$ \\ ${ }^{a}$ Institute of Nuclear and Particle Physics (INPP), NCSR Demokritos. \\ Agia Paraskevi, 15341, Athens-Greece \\ E-mail: panagiotis.assiouras@cern.ch
}

\begin{abstract}
In this work the results obtained with a calculation, in lower order, of backplane and interstrip capacitances in planar silicon microstrip sensors is presented. The method is based on a numerical solution of the 2D Laplace equation by partitioning the device in one dimension while keeping the other dimension continuous. The validity of the two-dimensional algorithm is checked through a comparison with experimental measurements and TCAD simulation performed on Multi Geometry Strip Sensors (MSSD).
\end{abstract}

The 28th International Workshop on Vertex Detectors - Vertex2019

13-18 October, 2019

Lopud, Croatia

${ }^{*}$ Speaker. 


\section{Introduction}

The most commonly used silicon sensors in High Energy Physics experiments are devices which have the one plane segmented to form strips (micro-strip sensors) or pixels (micro-pixel sensors). Parameters of great importance in the operation and the design of silicon sensors are the capacitances between adjacent strips or pixels and between the strips or pixels and the backplane. A numerical algorithm for solving the three dimensional Laplace's equation and calculating the capacitances of a pixel sensor, was presented in [1]. A similar algorithm has been implemented for calculating the capacitances of a micro-strip sensor by solving the Laplace's equation in two dimensions. These algorithms have been implemented within a program that can be used as a simulation tool for a fast approximate calculation of the capacitances. The results obtained using the two-dimensional numerical solution are compared with experimental results and TCAD simulations on Multi Geometry Strip Sensors (MSSD). The MSSD sensors were provided by the CMS group of Karlsruhe Institute of Technology.

\section{Two dimensional numerical solution of the Laplace's equation.}

In Figure 1 the capacitive network of a strip sensor with 5 strips is shown. The capacitances of interest are the strip to backplane capacitance $\left(C_{b}\right)$ and the interstrip capacitances between first neighbor $\left(C_{01}\right)$ and second neighbor strips $\left(C_{02}\right)$. The same method as in the three dimensional case adopted in [1] is followed, with the difference that in the strip sensor case the problem is reducible to two dimensions. The sensor is considered to be in a fully depleted state and it is free from thermally generated charged pairs of electron and holes. The strips are assumed to be infinitesimally small compared to the fully depleted region of the sensor. Also the volume and the surface of the sensor is free from static charges. Under real circumstances, charges exist inside the detector volume, those are stripped ions in the depleted regions and defects from contamination inside the material. However, they produce an electric field component which is independent of the biasing voltage. They simply add a voltage independent term in the expression of the strip charge which does not influence the calculation of the capacitance.

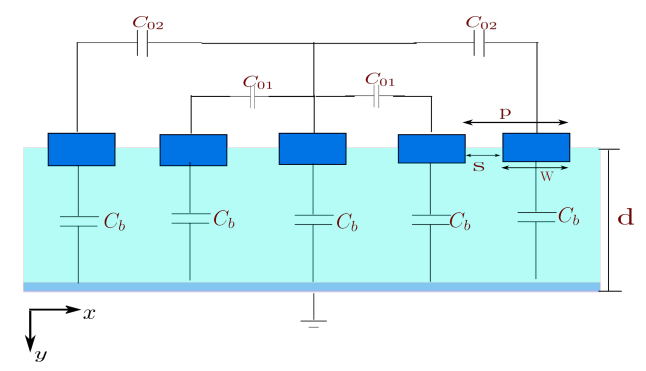

Figure 1: Schematic of the capacitive network of a micro-strip sensor with 5 strips.

To calculate the strip sensor capacitances the Poisson's equation is solved with normalized boundary conditions by setting $(V(x, y)=1)$ in the central strip, $(V(x, y)=0)$ to the adjacent strips and the back plane. In the areas not covered by strip, equation $\left(\varepsilon_{s i} E_{s i}(x, y)-\varepsilon_{a} E_{a}(x, y)=0\right)$ is used to keep the electric field at the interface continuous, where $\varepsilon_{s i}, \varepsilon_{a}$ are the dielectric constants and $E_{s i}(x, y), E_{a}(x, y)$ are the vertical components of the electric field in silicon and ambient space 
respectively, which is considered to be air in this model. The Poisson's equation is simplified to the Laplace's equation $\left(\nabla^{2} V(x, y)=0\right)$ by considering the simplifications above. A key characteristic of this method is that the axis that is parallel to the strip plane is discretized in finite elements, while the perpendicular axis is kept continuous. Subsequently by using Fourier transform, the two dimensional problem is reduced to one dimensional problem, equation 2.1.

$$
\frac{\partial^{2} V\left(k_{x}, y\right)}{\partial y^{2}}=k_{x}^{2} V\left(k_{x}, y\right)
$$

where $V\left(k_{x}, y\right)$ is the potential in Fourier space and $k_{x}$ is the corresponding coordinate in Fourier space. Then the differential equation 2.1 is solved by taking into account the boundary conditions which leads to equation 2.2 .

$$
V\left(k_{x}, y\right)=V\left(k_{x}\right) \frac{\sinh \left[k_{x}(d-y)\right]}{\sinh \left(k_{x} d\right)}
$$

By differentiating 2.2 with respect to $y$ axis equation 2.3 is derived which gives the electric field in Fourier space as a function of the potential in Fourier space.

$$
E\left(k_{x}, y\right)=k_{x} V\left(k_{x}\right) \frac{\cosh \left[k_{x}(d-y)\right]}{\sinh \left(k_{x} d\right)}
$$

The Laplace's equation is then solved in the Fourier space by using a self-consistent numerical method. First an initial guess of the potential is made $V^{i n i t}(x, 0)$ where for $(y=0)$ it corresponds to the strip plane. By using Fourier transform, with respect to $x$ axis the initial guess is transformed to the potential in Fourier space $V^{\text {init }}\left(k_{x}, 0\right)$. Then the vertical component of the electric field inside the sensor $E_{s i}^{i n i t}(x, 0)$ is calculated from 2.3 and by performing inverse Fourier transform. The vertical component of the electric field in the ambient space $E_{a}^{\text {init }}(x, 0)$ is calculated by equation 2.3 by setting $(d \rightarrow \infty)$ and by using inverse Fourier transform. Next the potential is redefined by boundary conditions. The values of the vertical component of the electric field in the ambient space $E_{a}^{\text {init }}(x, 0)$ are used for calculating the new values of the electric field inside the sensor $E_{s i}^{\text {init }}(x, 0)$, by using the boundary condition in the space that is not covered by strip. This gives a new estimation of the electric field inside the sensor $E_{\text {new }}(x, 0)$ and a new estimation of the potential $V_{\text {new }}(x, 0)$. The actual solution of the problem is assumed to be a linear combination of the new and the initial potential function. Then a check for convergence is made and if it has not been achieved the initial function is set equal to the new potential function, that has been derived from the linear combination. All the above steps are repeated through several cycles until convergence is reached.

Finally, once convergence has been achieved, the charges stored in each strip are calculated by integrating the charge density in the whole strip surface $\left(Q=\oiint\left(\varepsilon_{s i} E_{s i}-\varepsilon_{a} E_{a}\right) d S\right)$. Then the calculation of strip capacitances is made.

\section{Experimental data}

The validity of the calculations of the capacitances by using the two dimensional solution is checked with experimental measurements performed on MSSDs. The MSSDs contains 12 individual regions with 32 poly-silicon biased AC coupled strips on $n^{+} p$ configuration with pitches 
varying from 70 to $240 \mu \mathrm{m}$ resulting to width to pitch ratios varying from 0.133 to $0.321 \mu \mathrm{m}$. More details for these sensors can be found in ref [2].

The sensors that were tested in this work originating from 3 different wafers of float-zone silicon (FZ) and they have three different active thicknesses 120, 200, $320 \mu \mathrm{m}$. All the MSSD sensors that were tested in this work have been constructed with a p-stop isolation technique.

\section{TCAD Simulations.}

In Figure 2 the two dimensional structure for simulating the MSSD sensors with TCAD Sentaurus [3] is shown. The design resembles a perpendicular cut trough of the sensor to the strip plane. The final results are scaled to the actual sensor strip length. The structure consists of 5 strips with implant and aluminum widths corresponding to each MSSD region. The metal is extended a few $\mu \mathrm{m}$ in the interstrip space; this technique is called metal-overhang. In the space between two strips, two additional structures have been designed with high dose of p-implant resembling the actual p-stop structures.

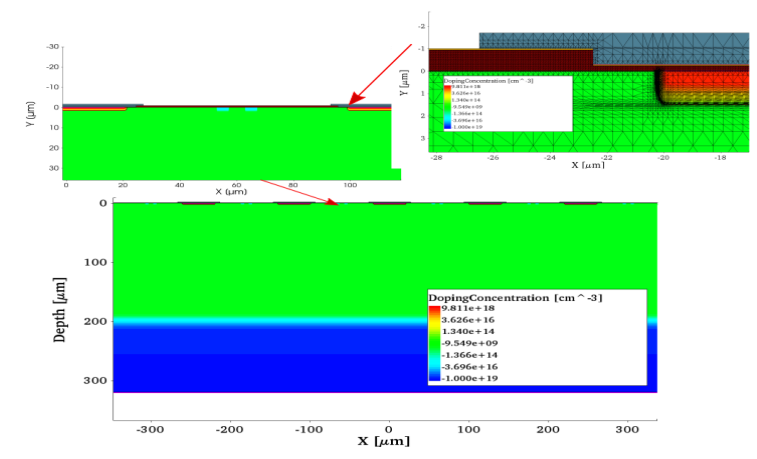

Figure 2: The simulated structure used for approximating the actual MSSD sensors.

The interstrip capacitances between two strips i and j for an AC coupled sensor are calculated, according to [4], with the following formula 4.1.

$$
C_{i n t}=C_{M i-M j}+C_{I i-I j}+C_{M i-I j}+C_{I i-M j}
$$

where $C_{M i-M j}$ is the capacitance between the metal of ith strip and the metal of jth strip, $C_{I i-I j}$ is the capacitance between the implant of ith strip and the implant of jth strip, $C_{M i-I j}$ and $C_{I i-M j}$ are the capacitances between adjacent metals and implants.

\section{Results.}

In this section a comparison is made between the calculated results by using our program with the experimental results and TCAD simulations on MSSD sensors.

\subsection{Comparison of the backplane capacitances.}

Histograms $3 \mathrm{a}, 3 \mathrm{~b}$ and $3 \mathrm{c}$ show the comparison for the backplane capacitances for the 3 MSSD sensors. The regions 3 and 12 from FZ200P sensor and 2 and 9 for the FZ320P sensor 
were damaged. The relative error of the simulated results $\left(C_{\text {sim }}\right)$ to the experimental values $\left(C_{\text {exp }}\right)$, defined as $\left(\left(\left|C_{\text {exp }}-C_{\text {sim }}\right| /\left|C_{\text {exp }}\right|\right) \%\right)$, of the numerical calculations has a mean value of $14 \%$ with a standard deviation of $7 \%$ while of the calculations made with TCAD has mean value of $12 \%$ and a standard deviation of $6 \%$.

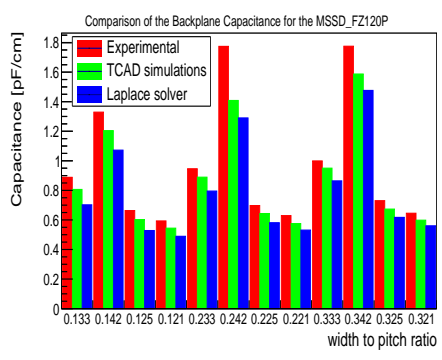

(a)

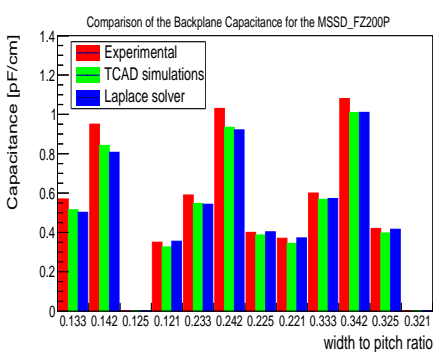

(b)

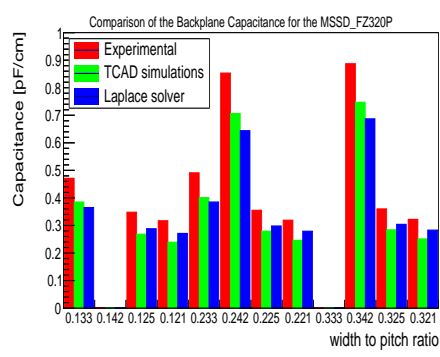

(c)

Figure 3: Comparison of the experimental results (red histogram) for the backplane capacitance with TCAD simulations (green histogram) and results from numerical simulations described in 2 (blue histogram) for 3a, FZ200P 3b and FZ320P 3c sensors.

\subsection{Comparison of the interstrip capacitances.}

Histograms 4a , 4b and 4c show the interstrip capacitances for the 3 MSSD sensors between the experimantal results, the TCAD simulations and the numerical calculations. The relative error of the simulated results to the experimental values of the numerical calculations has a mean value of $28 \%$ with standard deviation of $7 \%$ while of the calculations made with TCAD has a mean value of $11 \%$ and a standard deviation of $12 \%$.

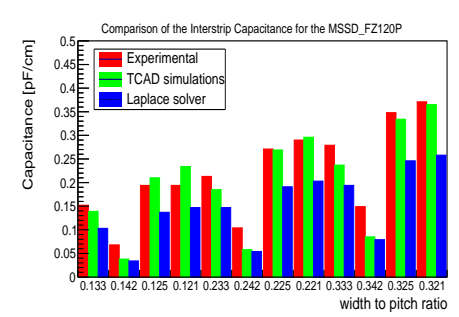

(a)

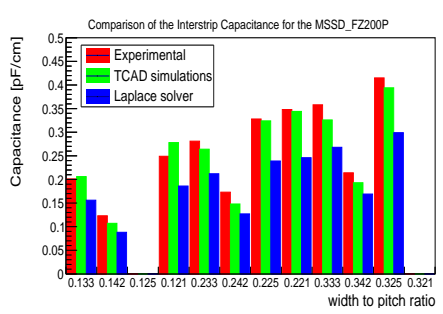

(b)

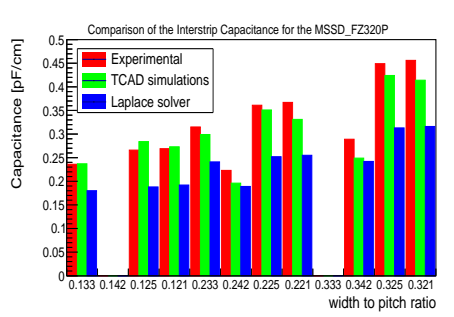

(c)

Figure 4: Comparison of the experimental results (red histogram) for the interstrip capacitance with TCAD simulations (green histogram) and results from numerical simulations described in 2 (blue histogram) for 4a, FZ200P 4b and FZ320P 4c sensors.

It must be noted that the numerical calculations are made for an ideal case where the sensor is free from static charges. Also it has no oxide and no aluminum contacts above the strip plane. Thus, it calculates only the capacitance between adjacent implants $C_{I i-I j}$. On the other hand, TCAD makes a more detailed simulation with more accurate physical models and also the interstrip capacitances are calculated by following equation 4.1 where the capacitances between adjacent aluminum $C_{M i-M j}$ and between adjacent aluminum and implants $C_{M i-I j}, C_{I i-M j}$ are taken into account. Also by changing the value of the dielectric constant of the ambient space to that of 
$\mathrm{SiO}_{2}$ doesn't improve the results, it slightly decrease the interstrip capacitance while the backplane remains the same.

\section{Conclusion}

The numerical solution of the Laplace's equation that was described in this work, approximate the experimental results and the TCAD simulations with a good accuracy. The relative error of the simulated results to the experimental values for calculating the backplane capacitance is (14 \pm 7$) \%$ and for calculating the interstrip capacitance is $(28 \pm 7) \%$.

In Table 1 the time that is needed to calculate the capacitances by using this program is shown for different degree of discretization. The calculations were performed with an 8 core processor at $3.70 \mathrm{GHz}$

\begin{tabular}{c|c}
\hline resolution & time $(\mathrm{sec})$ \\
\hline 1024 & 0.2 \\
16384 & 1.7 \\
65536 & 5.3 \\
524288 & 14.2 \\
\hline
\end{tabular}

Table 1: Calculation time for different degree of discretization

A program that implements the two dimensional numerical solution of the Laplace's equation that was described in this work can be used in order to provide a fast approximation of sensor capacitances for silicon strip sensors, before a more detailed simulation is made. This tool is foreseen to be implemented into a web-based application.

\section{Acknowledgments}

The authors of this work would like to thank the CMS group of Karlsruhe Institute of Technology for providing us the MSSD sensors. In addition, Patrick Asenov and Panagiotis Assiouras would like to acknowledge the support by the Hellenic Foundation for Research and Innovation, HFRI (Greece).

\section{References}

[1] S. Kavadias, K. Misiakos and D. Loukas, Calculation of pixel detector capacitances through three dimensional numerical solution of the Laplace equation, IEEE Transactions on Nuclear Science 41 (1994) 397.

[2] K.-H. Hoffmann, Campaign to identify the future cms tracker baseline, Nuclear Instruments and Methods in Physics Research Section A: Accelerators, Spectrometers, Detectors and Associated Equipment 658 (2011) 30 .

[3] https://www.synopsys.com/silicon/tcad.html, 2019.

[4] S. Chatterji, A. Bhardwaj, K. Ranjan, Namrata, A. K. Srivastava and R. Shivpuri, Analysis of interstrip capacitance of si microstrip detector using simulation approach, Solid-State Electronics 47 (2003) 1491 . 